\title{
Editorial
}

\section{Looking Beyond, Thinking Ahead: Use of Three-dimensional Patient Optimized Surgical Tools in Foot and Ankle Surgeries}

A once-shuttered warehouse is now a state-of-the art lab where new workers are mastering the $3 D$ printing that has the potential to revolutionize the way we make almost everything-Obama (State of Union Address, 2013).

Obama was making this in reference to potential return of high tech jobs back to US, but he was not far off when he called the technology a revolution that had the ability to transform every thing and indeed the medical field is embracing this with open arms. ${ }^{1}$ It has started to bring in a new realm of much awaited innovation into the sector that has always thrived on improving accuracy and achieving perfection.

In the foot and ankle surgery domain, planning accurate osteotomies, arthrodesis, arthroplasty and deformity corrections present unique challenge to foot and ankle surgeons. ${ }^{2,3}$ A thoughtful preoperative planning helps to be better prepared in terms of implant planning, anticipating untoward events and ensuring flawless execution. On the other hand, it is important that postoperatively assessment with regards to positioning and function be carried out.

The conventional ways to ensure preoperative planning and postoperative assessment was to use the radiographs. ${ }^{4}$ In complex cases, many surgeons used CT scan and the $3 \mathrm{D}$ reconstruction using specialized software. A recent addition to these existing techniques is prototyping or 3D modeling. ${ }^{5}$ In view of their ability to be specific to a patient, they are also called as patient optimized surgical tools or POST. The

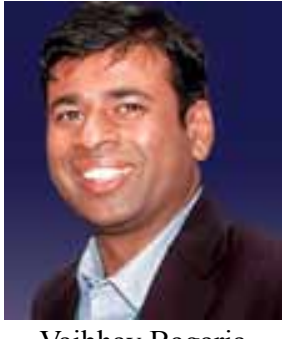

Vaibhav Bagaria

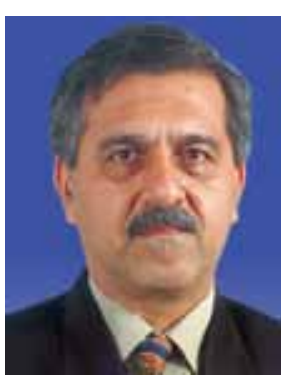

Mandeep S Dhillon technique involves creation of the life-sized model from the computed tomography (CT) digital imaging and communications in medicine (DICOM) images of the patient. ${ }^{6}$ The process involves converting the CT scan images into a machine printable language. These inputs are then transmitted to a 3D printing machine which using additive manufacturing technology creates a life-sized model (Fig. 1).

These models can then be used for surgical simulation preoperatively and as reference intraoperatively (Fig. 2). In complex cases, such as difficult primaries and revision condition, proper inventory planning, and also aid in deciding and sculpting bone grafts (Fig. 3). When done postoperatively, they can provide valuable information about the component positioning. The technique also provides data to develop patient specific instruments and implants similar to those popular in knee arthroplasty.

There are three types of 3D printing as follows:

- Fused deposit modeling: This is the most common technology available to surgeons and is also called as additive manufacturing. ${ }^{7}$ In this method, a spool of the thermoplastic substance is inserted into an extrusion head that heats this material into a semi-solid state. The extruder head then extrudes this semi-solid thermoplastic or similar material. Specialized software converts the axial image into a machine printable language that the machine presents layer by layer as a replica of the axial cuts (Fig. 4).

- Direct digital manufacturing: In this case, the device directly creates the end product. This printed product is ready to use as the machine prints the material that is fit for the end use. In case of medical field, it could be the implants made from innovative materials, like titanium, tantalum and also bioceramics, like hydroxylapatite and tri calcium phosphate. Having this technology at hand ensures a customized product for patient; this could be wedges, spacers, prosthesis or artificial bone

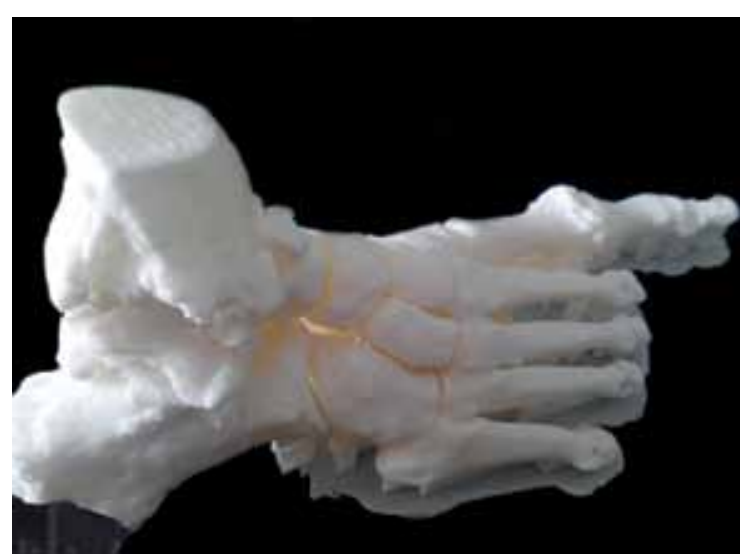

Fig. 1: A 3D printed model of foot based on the CT scan images of the foot

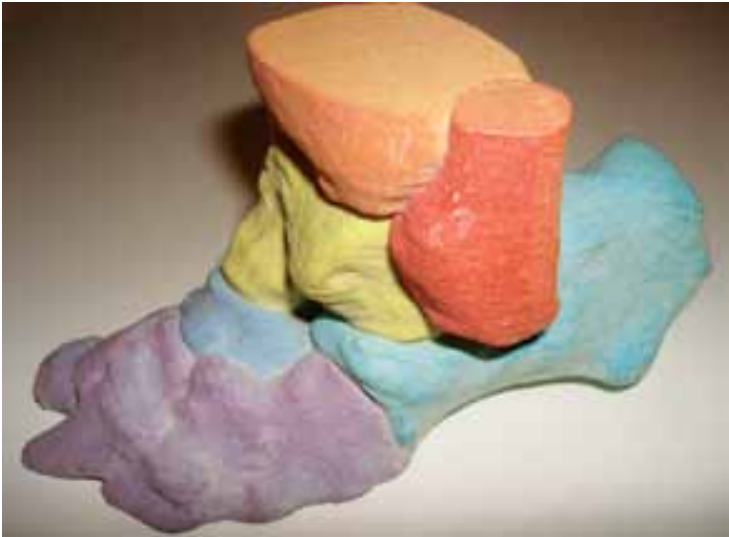

Fig. 2: Three-dimensional post as a teaching tool: A didactic model 


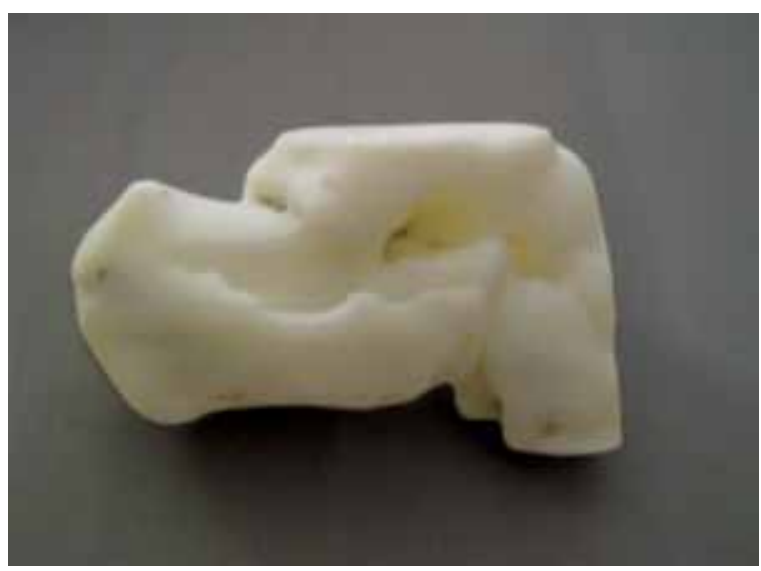

Fig. 3: A 3D model of a fracture calcaneum

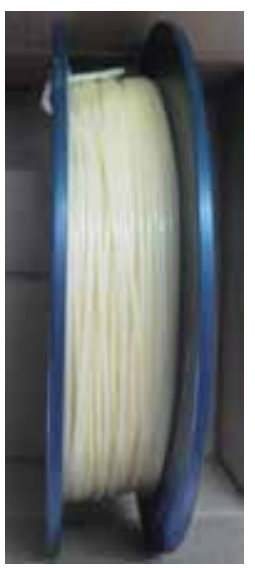

Fig. 4: Spool used for printing the model

for defects. The enthusiast for the technology believes that in future most prosthesis and implants available would be made using this technology.

- Polyjet: This technology helps to create highly precise parts and has added advantage because of its ability to combine different materials and different colors. In a way similar to the inkjet printers employed in day-to-day life, these printers can help to create models with over 1,000 physical properties and colors.

For foot and ankle surgeons, the 3D POST technology is especially helpful for conditions like triple fusion, wedge osteotomy, ankle arthroplasty planning, complex calcaneal fractures and deformity correction. While in fracture cases, surgeons can study the anatomy, pre-contour the plate and define the trajectory of implant and screws. In case of deformity correction, arthrodesis, osteotomies, and virtual simulation can be carried out. The initial experience has shown that it improves the accuracy, reduces surgical time, and decreases the intraoperative inventory and the perioperative blood loss.

Three-dimensional printing is likely to open a new avenue for accurate surgical planning and simulation for foot and ankle surgeons. These models can also prove to be a valuable teaching tool for students and residents. The future developments in the field can be in form of designing patient/disease specific instruments and implants.

Conflict of interest: The first author is co-founder of Medical 3D print company www.3dpost.in.

\section{REFERENCES}

1. Available at: http://edition.cnn.com/2013/02/13/tech/innovation/obama-3d-printing/

2. Siddiqui NA1, Lamm BM2. Digital planning for foot and ankle deformity correction: evans osteotomy. J Foot Ankle Surg 2014 NovDec;53(6):700-705.

3. Green C1, Fitzpatrick C, Fitz Patrick D, Stephens M, Quinlan W, Flavin R. Definition of coordinate system for three-dimensional data analysis in the foot and ankle. Foot Ankle Int 2011 Feb;32(2):193-199.

4. Devos Bevernage B1, Leemrijse T. Predictive value of radiographic measurements compared to clinical examination. Foot Ankle Int 2008 Feb;29(2):142-149.

5. Bagaria V, Deshpande S, Rasalkar DD, Kuthe A, Paunipagar BK. Use of rapid prototyping and three-dimensional reconstruction modeling in the management of complex fractures. Eur J Radiol 2011 Dec;80(3):814-820.

6. Frame M1, Huntley JS. Rapid prototyping in orthopaedic surgery: a user's guide. Scientific World Journal 2012;83857.

7. Basalah A1, Shanjani Y, Esmaeili S, Toyserkani E. Characterizations of additive manufactured porous titanium implants. J Biomed Mater Res B Appl Biomater 2012 Oct; 100.

Vaibhav Bagaria

Consultant and Surgeon Department of Joint Replacement and Sports Surgery Care Hospital and Origyn Clinic Nagpur, Maharashtra, India e-mail: bagariavaibhav@gmail.com

Mandeep S Dhillon

Professor and Head Department of Orthopedic Postgraduate Institute of Medical Education and Research, Chandigarh, India 